\title{
Stability of Grover's algorithm in respect to perturbations in quantum circuit
}

\author{
K. V. Gubaidullina, S. A. Chivilikhin \\ ITMO University, Kronverkskiy, 49, St. Petersburg, 197101, Russia \\ ksenya-gbd@yandex.ru, sergey.chivilikhin@gmail.com
}

PACS 03.67.-a

DOI 10.17586/2220-8054-2017-8-2-243-246

Grover's algorithm is a quantum algorithm for searching specified elements in an unsorted list. It has many valuable applications. The utilization of Grover's algorithm, to adapt it to accelerate the works of well-known classical algorithms, is very promising, and it is one of the fastest algorithms to solve such problems like global optimization and graph coloring. In this regard, it is very important to study the stability of the Grover's algorithm, to know how distortion of the circuit's elements affects on it results. This work presents the results of the simulation of Grover's algorithm, research of its stability with respect to perturbations of quantum logic circuit elements and its dependencies from the number of qubits, used in quantum circuit. Another part of this research was realized on IBM quantum processor and shows the stability of the 2-qubit Grover's algorithm.

Keywords: Grover's algorithm, quantum computations, quantum algorithms, IBM quantum processor.

Received: 23 January 2017

Revised: 4 March 2017

\section{Introduction}

Quantum computing uses a special kind of superposition, which allows exponentially many logical states simultaneously. This is a powerful feat, and no classical computer can achieve it. Quantum computer can solve many famous problems that classical computers cannot. For example, the problem of searching in unsorted list in classical algorithm actually reduces to sequential scan of $\mathrm{N}$ values, and uses on average N/2 steps. For a quantum computer, however, we can find the marked element use $\sqrt{\mathrm{N}}$ steps using Grover's algorithm [1].

The algorithm is performed on a search condition that is enclosed in a quantum oracle operator. This condition could be equal to some exact value, or we can for example find the minimum in some array. Such problem is relevant in searching for the optimal path on the graph [2] when it is necessary to find the shortest path between two vertices. In the classic case, many different methods of finding the minimum [3] could be used, and Dürr and Høyer in 1996 presented the method based on Grover's algorithm for finding the minimum with quantum enhancement [4].

We now describe the general stages of Grover's algorithm. At the initial time, the system is in the zero state. The Hadamard gate transforms the system into a state of superposition. After this transform, the followed iterative process consists of two functions: the first - phase rotation controlled by a search condition, which is enclosed in a quantum oracle operator; and the second - diffusion function makes a calculation of the amplitudes relative to its average. In the literature, this function is referred to as an inversion-about-average operator $[5,6]$.

The number of Grover's iterations must be strictly defined, otherwise the algorithm returns erroneous results. This quantity was calculated through formula (1) and it is enough to reach highest probability for algorithm with defined number of qubits $n$ :

$$
I=\frac{\pi}{4} \sqrt{2^{n}}
$$

The need to know in advance the number of values is a drawback of this algorithm - such situation in dealing with applications is quite rare. However, in the article [7,8] an algorithm was proposed for finding suitable search condition values that do not require an exact number of such values. Grovers algorithm is most effective with a large amount of data.

\section{Stability of multiqubit schemes}

The 3-qubit Grover's algorithm was simulated in article [9] and its stability to the effects of quantum logic circuit elements' perturbations was verified. Increasing perturbation values were shown to increase the probability of detecting the wrong state, but even the 3-qubit algorithm has good stability to the effects of quantum logic circuit element perturbations. The goal of this work was to simulate Grover's algorithm with more than 3 qubits, to study how doing this changes the probability of detection states and distortion impact. For example, a system 
with 11 qubits consists of 2048 states and needs 35 iterations, so this research helps one to know how the number of qubits affects the stability of Grover's algorithm.

The ways to affect the amplitude values of the states can be divided into two groups - light and strong deviations [9]. A histogram for the light deviations (Fig. 1) shows that the probability of detecting the marked state decreases with increased perturbation values.

Light deviations. Dependencies for schemes with 3, 6, 9, 11 qubits

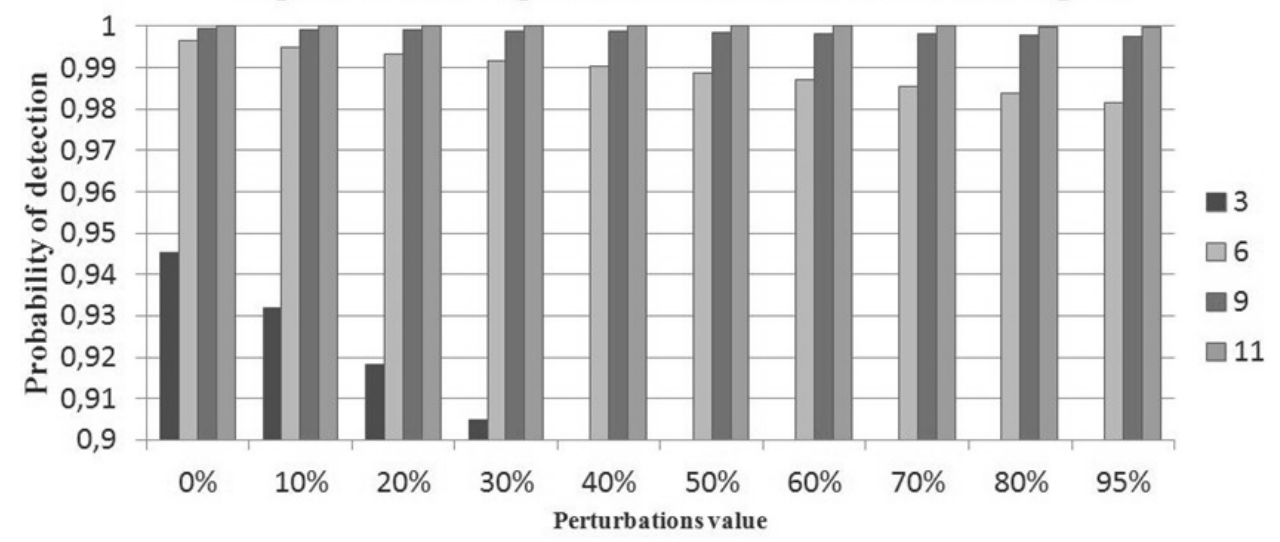

FIG. 1. Dependencies of detection probability and perturbation values for different number of qubits and light deviations

Correspondingly, the probability of detecting other (wrong) states increases, causing an increase in the frequency erroneous results. A larger number of qubits makes the algorithm more stable because of the number of necessary iterations that can be calculated by the formula (1) - a larger number of qubits requires a larger number of iterations. For example, 11 qubits with light deviations has extremely little distortion for very high value of perturbations.

Simulation of strong deviations (Fig. 2) is similar - the algorithm is more stable with a large number of qubits.

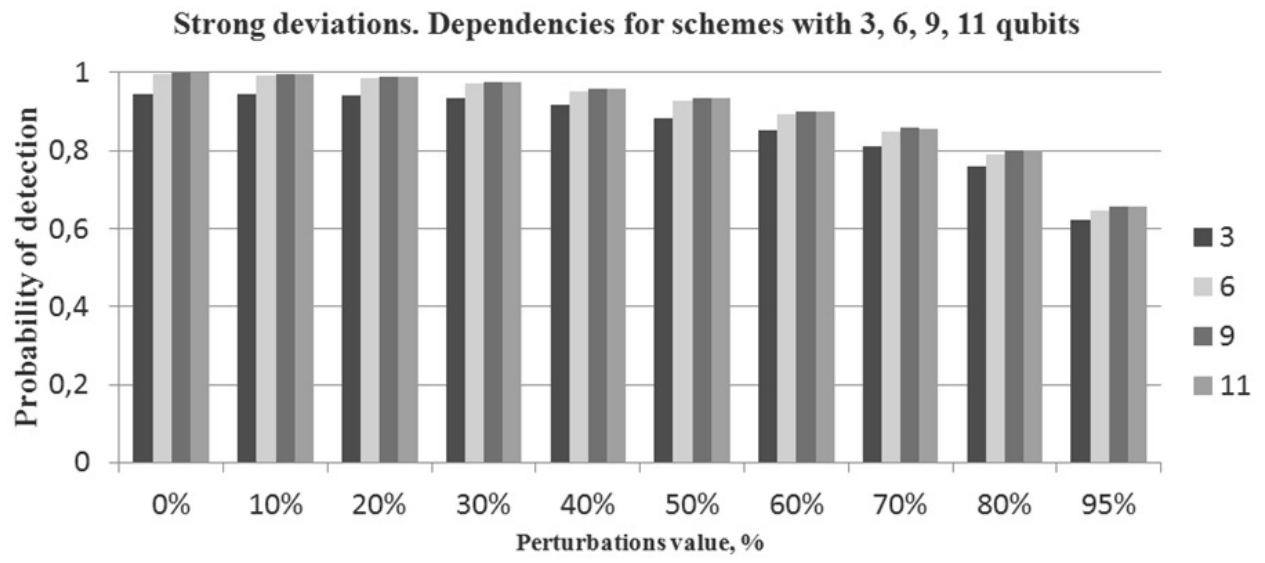

FIG. 2. Dependencies of detection probability and perturbation values for different numbers of qubits and strong deviations

In addition, it should be noted that the values of the probabilities for large systems is close to each other when error values increase. This is because the ideal values of the probabilities of these systems, when perturbation values are $0 \%$, are very close to one and respectively to each other. The stabilities of Grover's algorithm in these schemes are almost equal.

\section{IBM quantum experience}

IBM allows researchers to gain access to their 5-qubit quantum computer called IBM Quantum Experience (IBM QE). They use fixed-frequency superconducting transmon qubit [10] and the quantum processor itself is 
contained inside of a printed circuit board package. This package is mounted inside of a light-tight, magnetic-field shielding can, which sits at the coldest stage at the bottom of a dilution refrigerator, contained in IBM's Quantum Computing lab [11].

To perform the calculations, there is the Quantum Composer - a graphical user interface where the necessary quantum circuit can be created. Fig. 3 shows a representation of the platform with the scheme of Grover's algorithm for 2 qubits and marked state $|11\rangle$.

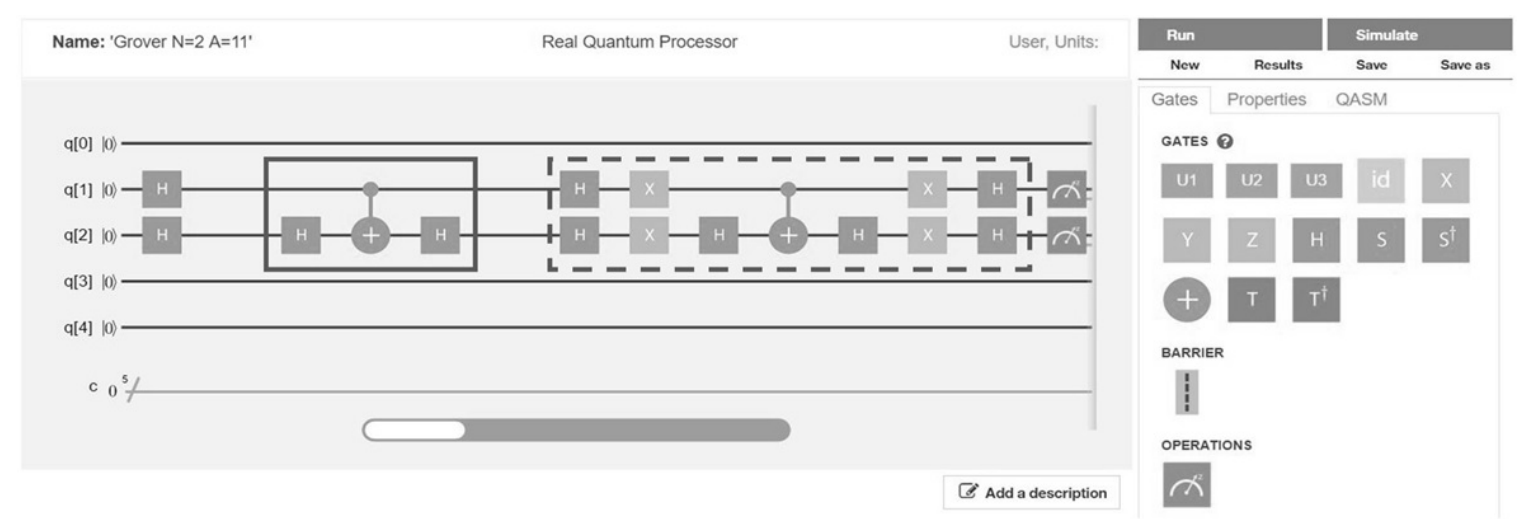

FIG. 3. Image of 5-qubit Quantum Composer and 2-qubit Grover's algorithm. Solid line box is a block of quantum oracle operator, and inside the dashed box - inversion-about-average operator

It is possible to run algorithms in simulation or on a real processor. Exploring the algorithm's running on a real quantum processor platform, its real stability with respect to circuit distortions can be seen and compared with theoretical calculations. There is also the opportunity to personally introduce distortions in the scheme collected by available gates and study their impact on the operation of the algorithm.

Topology of represented processor may allow the study only of the 2-qubit algorithm, so to compare the results in Fig. 4, the simulation of perturbation for 2-qubit Grover algorithm is presented. This is a histogram of average values for the strong and light deviation types.

\section{Stability of 2-qubit scheme. Probability of detecting marked state}

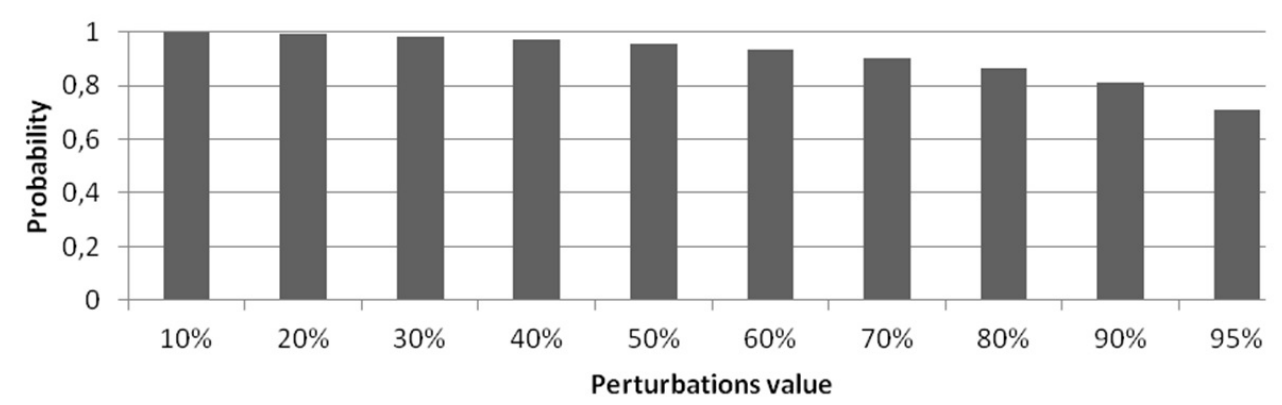

FIG. 4. Theoretical research of the distortion of 2-qubit Grover's algorithm

In an ideal 2-qubit circuit without any distortion, algorithm finds the right solution in 1 iteration in $100 \%$ of the cases. Due to the probabilistic nature of quantum algorithms and the existence of even the slightest noise in the circuit, which also affects the distribution of initial amplitudes, such a high proportion of correct answer detection is greatly reduced in the real computer. The algorithm was run by 8192 shots, and the results of every shot were considered to build a Fig. 5 - histogram of probability of detecting quantum states on the real quantum processor IBM.

The probability of detecting the correct state has decreased to $81.8 \%$. For theoretical simulation results in Fig. 4, this value corresponds to perturbations of the initial state amplitudes of about $80-85 \%$. Based on the fact that there is not only the distortion introduced by deviation of initial amplitudes, but other perturbations in the circuit, it can be concluded that this kind of deviation does not exceed $85 \%$. 
Probability of detecting states by IBM QE. Marked state $|11\rangle$

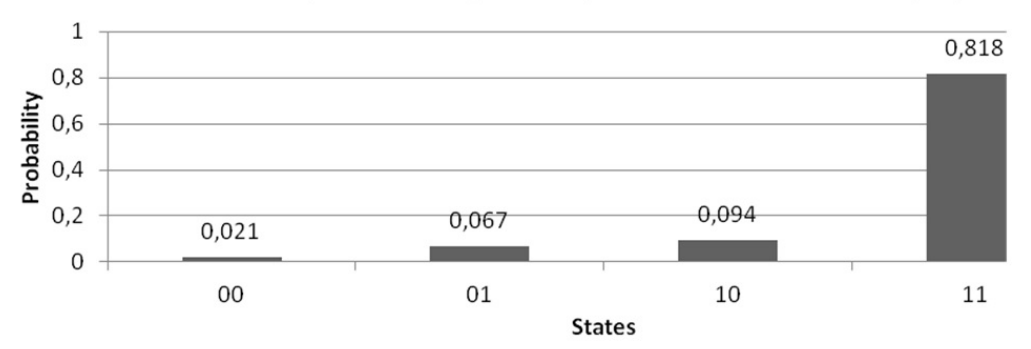

FIG. 5. Results for Grover's algorithm that have been computed by real quantum processor

\section{Conclusion}

By experimentation using the IBM quantum processor, computation of Grover's algorithm has been reviewed and compared with the theoretical results for a 2-qubit circuit. Theoretical simulation helps one to know how the number of qubits affects the stability of Grover's algorithm. It was shown that larger number of qubits and iterations makes the algorithm more stable. Another useful observation obtained from the results, is that stability of Grover's algorithm for schemes with large number of qubits is very similar. Thus, we can assume the stability of the algorithm on a larger number of qubits, which couldn't be calculated due to the insufficient computing power of classical computers.

\section{Acknowledgments}

This work was partially financially supported by the Government of the Russian Federation (grant 074U01).

\section{References}

[1] Grover L.K. A fast quantum mechanical algorithm for database search Proc. 28th Ann. ACM Symp. Theory of Computing (STOC) New York, ACM Press, 1996, P. 212-221.

[2] Aghaei M.R.S., Zukarnain Z.A., Mamat A., Zainuddin H. A hybrid algorithm for finding shortest path in network routing. J. Theoretical and Applied Inform. Technol, 2009, P. 360-365.

[3] Koistinen O.-P., Maras E., Vehtari A., J'onsson H. Minimum energy path calculations with Gaussian process regression. Nanosystems: Physics, Chemistry, Mathematics, 2016, 7(6), P. 925-935.

[4] Dürr C., Høyer P. A quantum algorithm for finding the minimum arXiv preprint quant-ph/9607014, 1996.

[5] Linington I.E., Ivanov P.A., Vitanov N.V. Quantum search in a nonclassical database of trapped ions. Phys. Rev. A, 2009 , 79, P. 012322.

[6] Nakahara M., Ohmi T. Quantum Computing: From Linear Algebra to Physical Realizations. CRC Press, 2008.

[7] Boyer M., Brassard G., Høyer P., Tapp A. Tight bounds on quantum searching. arXiv preprint quant-ph/9605034, 1996.

[8] Toyama F.M., Dijk W., Nogami Y. Quantum search with certainty based on modified Grover algorithms: optimum choice of parameters. Quantum Inf Process, 2013, 12, P. 1897-1914.

[9] Gubaidullina K.V., Chivilikhin S.A. Theoretical research of the distortion of quantum circuit in Grover's algorithm Journal of Physics: Conference Series, 2016, 735, P. 012074-1-012074-6.

[10] Koch J., Yu T.M., et al. Charge-insensitive qubit design derived from the Cooper pair box. Phys. Rev. A, 2007 , 76, P. 042319.

[11] IBM Research Quantum Experience. URL: www.research.ibm.com/quantum. 